\title{
Critical exponent for damped wave equations with nonlinear memory
}

\author{
Ahmad Z. FINO \\ ${ }^{a}$ Laboratoire de mathématiques appliquées, UMR CNRS 5142, Université de Pau et des Pays de l'Adour, 64000 Pau, France \\ ${ }^{b}$ LaMA-Liban, Lebanese University, P.O. Box 37 Tripoli, Lebanon
}

\begin{abstract}
We consider the Cauchy problem in $\mathbb{R}^{n}, n \geq 1$, for a semilinear damped wave equation with nonlinear memory. Global existence and asymptotic behavior as $t \rightarrow \infty$ of small data solutions have been established in the case when $1 \leq n \leq 3$. Moreover, we derive a blow-up result under some positive data in any dimensional space.

Keywords: Nonlinear damped wave equation, Global existence, Blow-up, Critical exponent, Large time asymptotic behavior

2010 MSC: 35L15, 35L70, 35B33, 34B44
\end{abstract}

\section{Introduction}

This paper concerns with the Cauchy problem for the damped wave equation with nonlinear memory

$$
\begin{cases}u_{t t}-\Delta u+u_{t}=\int_{0}^{t}(t-s)^{-\gamma}|u(s)|^{p} d s & t>0, x \in \mathbb{R}^{n}, \\ u(0, x)=u_{0}(x), \quad u_{t}(0, x)=u_{1}(x) & x \in \mathbb{R}^{n},\end{cases}
$$

where the unknown function $u$ is real-valued, $n \geq 1,0<\gamma<1$ and $p>1$. Throughout this paper, we assume that

$$
\left(u_{0}, u_{1}\right) \in H^{1}\left(\mathbb{R}^{n}\right) \times L^{2}\left(\mathbb{R}^{n}\right)
$$

and

$$
\operatorname{supp} u_{i} \subset B(K):=\left\{x \in \mathbb{R}^{n}:|x|<K\right\}, \quad K>0, i=0,1 .
$$

For the simplicity of notations, $\|\cdot\|_{q}$ and $\|\cdot\|_{H^{1}}(1 \leq q \leq \infty)$ stand for the usual $L^{q}\left(\mathbb{R}^{n}\right)$-norm and $H^{1}\left(\mathbb{R}^{n}\right)$-norm, respectively.

The nonlinear nonlocal term can be considered as an approximation of the classical semilinear damped wave equation

since the limit

$$
u_{t t}-\Delta u+u_{t}=|u(t)|^{p}
$$

$$
\lim _{\gamma \rightarrow 1} \frac{1}{\Gamma(1-\gamma)} s_{+}^{-\gamma}=\delta(s)
$$

exists in distribution sense, where $\Gamma$ is the Euler gamma function.

It is clear that this nonlinear term involves memory type selfinteraction and can be considered as Riemann-Liouville integral operator

$$
J_{a \mid t}^{\alpha} g(t):=\frac{1}{\Gamma(\alpha)} \int_{a}^{t}(t-s)^{\alpha-1} g(s) d s
$$

Email address: ahmad.fino01@gmail.com (Ahmad Z. FINO) 
introduced with $a=-\infty$ by Liouville in 1832 and with $a=0$ by Riemann in 1876 (see Chapter V in [4]). Therefore, (1.1) takes the form

$$
u_{t t}-\Delta u+u_{t}=J_{0 \mid t}^{\alpha}\left(|u|^{p}\right)(t)
$$

where $\alpha=1-\gamma$.

In recent years, questions of global existence and blow-up of solutions for nonlinear hyperbolic equations with a damping term have been studied by many mathematicians, see $[11,12,17,22,24]$ and the references therein. To focus on our motivation, we shall mention below only some results related to Todorova and Yordanov [24]. For the Cauchy problem for the semilinear damped wave equation with the forcing term

$$
u_{t t}-\Delta u+u_{t}=|u|^{p}, \quad u(0)=u_{0}, \quad u_{t}(0)=u_{1},
$$

it has been conjectured that the damped wave equation has the diffuse structure as $t \rightarrow \infty$ (see e.g. [1, 16]). This suggests that problem (1.5) should have $p_{c}(n):=1+2 / n$ as critical exponent which is called the Fujita exponent named after Fujita [8], in general space dimension. Indeed, Todorova and Yordanov [24] have showed that the critical exponent is exactly $p_{c}(n)$, that is, if $p>p_{c}(n)$ then all small initial data solutions of (1.5) are global, while if $1<p<$ $p_{c}(n)$ then all solutions of (1.5) with initial data having positive average value blow-up in finite time regardless of the smallness of the initial data. Moreover, they showed that in the case of $p>p_{c}(n)$, the support of the solution of (1.5) is strongly suppressed by the damping, so that the solution is concentrated in a ball much smaller than $|x|<t+K$, namely

$$
\|D u(t, \cdot)\|_{L^{2}\left(\mathbb{R}^{n} \backslash B\left(t^{1 / 2+\delta}\right)\right)}=O\left(e^{-t^{2 \delta / 4}}\right), \quad \text { as } t \rightarrow \infty,
$$

where $D:=\left(\partial_{t}, \nabla_{x}\right)$. Furthermore, they proved that the total energy of the solutions of (1.5) decays at the rate of the linear equation, namely

$$
\|D u(t, \cdot)\|_{L^{2}\left(\mathbb{R}^{n}\right)}=O\left(t^{-n / 4-1 / 2}\right), \quad \text { as } t \rightarrow \infty .
$$

Our goal is to apply the above properties founded by Todorova and Yordanov to our problem (1.1) with the same assumptions on the initial data. The method used to prove the global existence is inspired from the weighted energy method developed in [24]. On the other hand, the test function method (see [5, 6, 7, 14, 15, 18, 19, 25] and the references therein) is the key to prove the blow-up result. We denote that our global existence and asymptotic behavior as $t \rightarrow \infty$ for small data solutions are obtained in the case when $1 \leq n \leq 3$, due to the nonlocal in time nonlinearity. While the blow-up result is done in any dimensional space. Let us present our main results.

First, the following local well-posedness result is needed.

Proposition 1. Let $1<p \leq n /(n-2)$ for $n \geq 3$, and $p \in(1, \infty)$ for $n=1,2$. Under the assumptions (1.2)-(1.3) and $\gamma \in(0,1)$, the problem (1.1) possesses a unique maximal mild solution $u$, i.e. satisfies the integral equation (3.22) below, such that

$$
u \in C\left(\left[0, T_{\max }\right), H^{1}\left(\mathbb{R}^{n}\right)\right) \cap C^{1}\left(\left[0, T_{\max }\right), L^{2}\left(\mathbb{R}^{n}\right)\right),
$$

where $0<T_{\max } \leq \infty$. Moreover, $u(t, \cdot)$ is supported in the ball $B(t+K)$. In addition:

$$
\text { either } T_{\max }=\infty \text { or else } T_{\max }<\infty \text { and }\|u(t)\|_{H^{1}}+\left\|u_{t}(t)\right\|_{2} \rightarrow \infty \text { as } t \rightarrow T_{\max } .
$$

Remark 1. We say that $u$ is a global solution of (1.1) if $T_{\max }=\infty$, while in the case of $T_{\max }<\infty$, we say that $u$ blows up in finite time.

Now, set

$$
p_{\gamma}:=1+\frac{2(2-\gamma)}{(n-2+2 \gamma)_{+}}, \quad p_{1}:=1+\frac{2(3-2 \gamma)}{(n-2+2 \gamma)_{+}}, \quad p_{2}:=1+\frac{4(3-2 \gamma)}{(n-4+4 \gamma)_{+}} \quad \text { and } \quad p_{3}:=1+\frac{n+2(5-4 \gamma)}{(n-2+4 \gamma)_{+}} .
$$

As

$$
\left(p_{\gamma}=n /(n-2)=1 / \gamma\right) \Longleftrightarrow(\gamma=(n-2) / n),
$$


this imply, in the case when $(n-2) / n<\gamma$, that $p_{\gamma}=\max \left\{1 / \gamma ; p_{\gamma}\right\}<n /(n-2)$. Moreover, $p_{\gamma}<\min _{1 \leq n \leq 3}\left(p_{n}\right)$. We note that

$$
p_{\gamma}, p_{1} \rightarrow 1+2 / n=p_{c}(n), \quad p_{2} \rightarrow(2 \gamma+1) /(2 \gamma-1)>p_{c}(2) \quad \text { and } \quad p_{3} \rightarrow 2>p_{c}(3) \quad \text { as } \gamma \rightarrow 1 .
$$

Our global existence result is the following

Theorem 1. Let $1 \leq n \leq 3, p>1, \gamma \in(1 / 2,1)$ for $n=1,2$ and $\gamma \in(11 / 16,1)$ for $n=3$. Assume that the initial data satisfy (1.2)-(1.3) such that $\left\|u_{0}\right\|_{H^{1}}+\left\|u_{1}\right\|_{L^{2}}$ is sufficiently small. If $p_{n}<p$ then the problem (1.1) admits a unique global mild solution

$$
u \in C\left([0, \infty), H^{1}\left(\mathbb{R}^{n}\right)\right) \cap C^{1}\left([0, \infty), L^{2}\left(\mathbb{R}^{n}\right)\right) .
$$

Note that, the requirement $\gamma \in(11 / 16,1)$ is just to assure that $p_{3}<n /(n-2)$ when $n=3$.

The second result is the finite time blow-up of the solution under some positive data which shows that the assumption on the exponent in the above theorem (for $n=1$ and $\gamma \rightarrow 1$ ) is critical and it is exactly the same critical exponent to the semilinear heat equation $u_{t}-\Delta u=|u|^{p}$. Moreover, we conjecture that $p_{1}$ will be the critical exponent of (1.1) which is the critical one to the corresponding semilinear heat equation $u_{t}-\Delta u=\int_{0}^{t}(t-s)^{-\gamma}|u(s)|^{p} d s$ founded by Cazenave, Dickstein and Weissler [2] and Fino and Kirane [7].

\section{Theorem 2.}

i) Let $1<p \leq n /(n-2)$ for $n \geq 3$, and $p \in(1, \infty)$ for $n=1,2$. Assume that $(n-2) / n<\gamma<1$ and $\left(u_{0}, u_{1}\right)$ satisfy (1.2)-(1.3) such that

$$
\int_{\mathbb{R}^{n}} u_{i}(x) d x>0, \quad i=0,1 .
$$

If $p \leq p_{\gamma}$, then the mild solution of the problem (1.1) blows up in finite time.

ii) Let $n \geq 3$ and $1<p \leq n /(n-2)$. Assume that $\gamma \leq(n-2) / n$ and $\left(u_{0}, u_{1}\right)$ satisfy (1.2) and (1.7), then the mild solution of the problem (1.1) blows up in finite time.

As the by-product of our analysis in Theorem 1, we have the following result concerning the asymptotic behavior as $t \rightarrow \infty$ of solutions.

Theorem 3. Under the assumptions of Theorem 1 the asymptotic behavior of the small data global solution u of (1.1) is given by

$$
\|D u(t, \cdot)\|_{L^{2}\left(\mathbb{R}^{n} \backslash B\left(t^{1 / 2+\delta}\right)\right)}=O\left(e^{-t^{2 \delta / 4}}\right), \quad t \rightarrow \infty,
$$

that is the solution decays exponentially outside every ball $B\left(t^{1 / 2+\delta}\right), \delta>0$. Moreover, the total energy satisfies

$$
\|D u(t, \cdot)\|_{L^{2}\left(\mathbb{R}^{n}\right)}=O\left(t^{-n / 4+1 / 2-\gamma}\right), \quad t \rightarrow \infty,
$$

for $n=1$,

for $n=2$ and

$$
\|D u(t, \cdot)\|_{L^{2}\left(\mathbb{R}^{n}\right)}=O\left(t^{1 / 2-\gamma}\right), \quad t \rightarrow \infty
$$

for $n=3$.

$$
\|D u(t, \cdot)\|_{L^{2}\left(\mathbb{R}^{n}\right)}=O\left(t^{-\gamma}\right), \quad t \rightarrow \infty,
$$

As we have seen, we are restricted ourselves in the case of compactly supported data. This restriction leads us to the finite propagation speed property of the wave which plays an important role in the proof of the global solvability. The blow-up result and the local existence theorem could be proved removing the requirement for the compactness assumptions on the support of the initial data. For the global existence without assuming the compactness of support on the initial data, we refer the reader to $[9,10,11,20,21]$ where we have to take $u_{0} \in H^{1}\left(\mathbb{R}^{n}\right) \cap L^{1}\left(\mathbb{R}^{n}\right)$ and $u_{1} \in L^{2}\left(\mathbb{R}^{n}\right) \cap L^{1}\left(\mathbb{R}^{n}\right)$.

Remark 2. It is still open to show corresponding global existence of solutions, with small initial data, for $p_{\gamma}<p<$ $p_{n}(1 \leq n \leq 3)$ and for $p_{\gamma}<p(n \geq 4)$. 
This paper is organized as follows: in Section 2, we present some definitions and properties concerning the fractional integrals and derivatives. Section 3 contains the proofs of the global existence theorem (Theorem 11) and the asymptotic behavior of solution (Theorem 3). Section 4 is devoted to the proof of the blow-up result (Theorem 2). Finally, to make this paper self-contained, we shall sketch the proof of the local existence of solution (Proposition 1 ) in Appendix A

\section{Preliminaries}

In this section, we give some preliminary properties on the fractional integrals and fractional derivatives that will be used in the proof of Theorem 2 .

If $A C[0, T]$ is the space of all functions which are absolutely continuous on $[0, T]$ with $0<T<\infty$, then, for $f \in A C[0, T]$, the left-handed and right-handed Riemann-Liouville fractional derivatives $D_{0 \mid t}^{\alpha} f(t)$ and $D_{t \mid T}^{\alpha} f(t)$ of order $\alpha \in(0,1)$ are defined by

$$
D_{0 \mid t}^{\alpha} f(t):=\partial_{t} J_{0 \mid t}^{1-\alpha} f(t) \quad \text { and } \quad D_{t \mid T}^{\alpha} f(t):=-\frac{1}{\Gamma(1-\alpha)} \partial_{t} \int_{t}^{T}(s-t)^{-\alpha} f(s) d s, \quad t \in[0, T],
$$

where

$$
J_{0 \mid t}^{\alpha} g(t):=\frac{1}{\Gamma(\alpha)} \int_{0}^{t}(t-s)^{\alpha-1} g(s) d s
$$

is the Riemann-Liouville fractional integral, for all $g \in L^{q}(0, T)(1 \leq q \leq \infty)$. We refer the reader to [13] for the definitions above. Furthermore, for every $f, g \in C([0, T])$ such that $D_{0 \mid t}^{\alpha} f(t), D_{t \mid T}^{\alpha} g(t)$ exist and are continuous, for all $t \in[0, T], 0<\alpha<1$, we have the formula of integration by parts (see (2.64) p. 46 in [23])

$$
\int_{0}^{T}\left(D_{0 \mid t}^{\alpha} f\right)(t) g(t) d t=\int_{0}^{T} f(t)\left(D_{t \mid T}^{\alpha} g\right)(t) d t
$$

Note also that, for all $f \in A C^{n+1}[0, T]$ and all integer $n \geq 0$, we have (see (2.2.30) in [13])

$$
(-1)^{n} \partial_{t}^{n} \cdot D_{t \mid T}^{\alpha} f=D_{t \mid T}^{n+\alpha} f,
$$

where

$$
A C^{n+1}[0, T]:=\left\{f:[0, T] \rightarrow \mathbb{R} \text { and } \partial_{t}^{n} f \in A C[0, T]\right\}
$$

and $\partial_{t}^{n}$ is the usual $n$ times derivative. Moreover, for all $1 \leq q \leq \infty$, the following formula (see [13, Lemma 2.4 p.74])

$$
D_{0 \mid t}^{\alpha} J_{0 \mid t}^{\alpha}=I d_{L^{q}(0, T)}
$$

holds almost everywhere on $[0, T]$.

In the proof of Theorem 2 , the following results are useful: if $w_{1}(t)=(1-t / T)_{+}^{\sigma}, t \geq 0, T>0, \sigma \gg 1$, then

$$
D_{t \mid T}^{\alpha} w_{1}(t)=C T^{-\sigma}(T-t)_{+}^{\sigma-\alpha}, \quad D_{t \mid T}^{\alpha+1} w_{1}(t)=C T^{-\sigma}(T-t)_{+}^{\sigma-\alpha-1}, \quad D_{t \mid T}^{\alpha+2} w_{1}(t)=C T^{-\sigma}(T-t)_{+}^{\sigma-\alpha-2},
$$

for all $\alpha \in(0,1)$; so

$$
\left(D_{t \mid T}^{\alpha} w_{1}\right)(T)=0, \quad\left(D_{t \mid T}^{\alpha} w_{1}\right)(0)=C T^{-\alpha}, \quad\left(D_{t \mid T}^{\alpha+1} w_{1}\right)(T)=0 \quad \text { and } \quad\left(D_{t \mid T}^{\alpha+1} w_{1}\right)(0)=C T^{-\alpha-1} .
$$

For the proof of this results, see [5, Preliminaries]. Furthermore, the following lemma is useful to prove Theorem 1

Lemma 1. ([3, Lemma 4.1]) Suppose that $0 \leq \theta<1, a \geq 0$ and $b \geq 0$. Then there exists a constant $C>0$ depending only on $a, b$ and $\theta$ such that fot all $t>0$,

$$
\int_{0}^{t}(t-\tau)^{-\theta}(1+t-\tau)^{-a}(1+\tau)^{-b} d \tau \leq \begin{cases}C(1+t)^{-\min (a+\theta, b)} & \text { if } \max (a+\theta, b)>1 \\ C(1+t)^{-\min (a+\theta, b)} \ln (2+t) & \text { if } \max (a+\theta, b)=1 \\ C(1+t)^{1-a-\theta-b} & \text { if } \max (a+\theta, b)<1 .\end{cases}
$$

Throughout this paper, positive constants will be denoted by $C$ and will change from line to line. 


\section{Global existence and asymptotic behavior}

In view of the Proposition 1, global existence of a solution follows from the boundedness of its energy at all times. To obtain such a priori estimates, we shall proceed our proof based on the weighted energy method recently developed in Todorova and Yordanov [24]. We begin by defining

$$
\psi(x, t)=\frac{1}{2}\left(t+K-\sqrt{(t+K)^{2}-|x|^{2}}\right), \quad|x|<t+K .
$$

It is easily checked that $\psi_{t}<0$,

$$
0<\psi(x, t)<\frac{K}{2}
$$

and, since

$$
\sqrt{(t+K)^{2}-|x|^{2}} \leq t+K-|x|^{2} /[2(t+K)],
$$

the function $\psi$ satisfies the inequality

$$
\psi(x, t) \geq \frac{|x|^{2}}{4(t+K)} .
$$

Proof of Theorem 1. Let $u$ be the local solution of the problem (1.1) in $\left[0, T_{\max }\right)$. Let us introduce the energy functional

$$
W(t):=(1+t)^{j}\|D u(t, \cdot)\|_{2},
$$

where

$$
j:=n / 4-1 / 2+\gamma \quad(n=1), \quad j:=\gamma-1 / 2 \quad(n=2) \quad \text { and } \quad j:=\gamma \quad(n=3) .
$$

We will show that $W(t) \leq C I_{0}$, where $I_{0}:=\left\|u_{0}\right\|_{H^{1}}+\left\|u_{1}\right\|_{2}$ is small enough. This not only gives the global existence but also shows that, for $n=1$ and $\gamma \rightarrow 1$, the solution decays at least as fast as that of the linear part $u_{t t}-\Delta u+u_{t}=0$. For the rate of the linear problem, see (3.23) below.

The estimate (3.11) will be done by the following lemmas.

Lemma 2. Let $1 \leq n \leq 3, \gamma \in(1 / 2,1)$ for $n=1,2$ and $\gamma \in(11 / 16,1)$ for $n=3$. For all $\delta>0$ and all $t \in\left[0, T_{\max }\right)$, the following weighted energy estimate holds

$$
(1+t)^{j}\|D u(t, \cdot)\|_{2} \leq C I_{0}+C\left(\max _{[0, t]}(1+\tau)^{\beta}\left\|e^{\delta \psi(\tau, \cdot)} u(\tau, \cdot)\right\|_{2 p}\right)^{p},
$$

where $\beta>n / 4 p+(2-\gamma) / p$ for $n=1,3$ and $\beta>(2-\gamma) / p$ for $n=2$.

Lemma 3. ([24, Proposition 2.4]) Let $\theta(q)=n(1 / 2-1 / q)$ and $0 \leq \theta(q) \leq 1$, and let $0<\sigma \leq 1$. If $u \in H^{1}\left(\mathbb{R}^{n}\right)$ with suppu $\subset B(t+K), t \geq 0$. Then

$$
\left\|e^{\sigma \psi(t,)} u\right\|_{q} \leq C_{K}(1+t)^{(1-\theta(q)) / 2}\|\nabla u\|_{2}^{1-\sigma}\left\|e^{\psi(t, \cdot)} \nabla u\right\|_{2}^{\sigma}, .
$$

where $\psi(t, x)$ is the weight function from 3.8 .

We postpone the proof of Lemma2 to the end of this section.

It follows from Lemma 2 that

$$
W(t) \leq C I_{0}+C\left(\max _{[0, t]}(1+\tau)^{\beta}\left\|e^{\delta \psi(\tau, \cdot)} u(\tau, \cdot)\right\|_{2 p}\right)^{p} .
$$

On the other hand, Lemma 3 with $q=2 p$ and $\sigma=\delta \leq 1$ gives

$$
\begin{aligned}
\left\|e^{\delta \psi(\tau, \cdot)} u(\tau, \cdot)\right\|_{2 p} & \leq C(1+\tau)^{1-\theta(2 p)) / 2}\|\nabla u\|_{2}^{1-\delta}\left\|e^{\psi(t, \cdot)} \nabla u\right\|_{2}^{\delta} \\
& \leq C(1+\tau)^{(1-\theta(2 p)) / 2-j} W(\tau),
\end{aligned}
$$

where we have used (3.9).

Using (3.15), we obtain from 3.14

$$
W(t) \leq C I_{0}+C\left(\max _{[0, t]}(1+\tau)^{\beta+(1-\theta(2 p)) / 2-j} W(\tau)\right)^{p} .
$$


Set $\beta=n / 4 p+(2-\gamma) / p+v$ for $n=1,3$ and $\beta=(2-\gamma) / p+v$ for $n=2, v>0$, then if we compute the exponent of $(\tau+1)$ in the right side of (3.16), we obtain

$$
\beta+(1-\theta(2 p)) / 2-j= \begin{cases}v-\frac{n}{2 p}[p(1-2(1-\gamma) / n)-1-2(2-\gamma) / n], & \text { if } n=1, \\ v-\frac{n}{4 p}[p(1-4(1-\gamma) / n)-1-4(2-\gamma) / n], & \text { if } n=2, \\ v-\frac{n}{4 p}[p(1+2(2 \gamma-1) / n)-2-4(2-\gamma) / n], & \text { if } n=3 .\end{cases}
$$

As $p>p_{n}$, we deduce, choosing $v$ small enough, that the quantities in (3.17) are negative. Hence, we can rewrite (3.16) like

$$
\max _{[0, t]} W(\tau) \leq C I_{0}+C\left(\max _{[0, t]} W(\tau)\right)^{p} .
$$

Now, write $I_{0}=\left\|u_{0}\right\|_{H^{1}}+\left\|u_{1}\right\|_{2}=C \varepsilon$, for small $\varepsilon>0$ which is determined later, and put

$$
T^{*}=\sup \{t \geq 0: W(t) \leq 2 C \varepsilon\} .
$$

Then, (3.18) implies $W(t) \leq C \varepsilon+C \varepsilon^{p}$. Therefore, taking small $\varepsilon$ such that $C \varepsilon+C \varepsilon^{p}<2 C \varepsilon$ we conclude that $T^{*}=\infty$ ( For details we refer the reader to [11, Proposition 2.1] and [22, Proposition 2.1]), i.e.

$$
W(t)=(1+t)^{j}\|D u(t, \cdot)\|_{2} \leq C \varepsilon, \quad t \geq 0 .
$$

Thus we have completed the proof of Theorem 1 .

Proof of Theorem 3, The estimate (1.9) - (1.11) follows directly from (3.19). Next, it follows from inequality (3.9)-(3.10) and estimate (3.19) that

$$
C \varepsilon \geq\left\|e^{\psi(t, \cdot)} D u(t, \cdot)\right\|_{L^{2}\left(\mathbb{R}^{n}\right)} \geq\left\|e^{|\cdot|^{2} / 4(t+K)} D u(t, \cdot)\right\|_{L^{2}\left(\mathbb{R}^{n} \backslash B\left(t^{1 / 2+\delta}\right)\right)} \geq e^{t^{1+2 \delta} / 4(t+K)}\|D u(t, \cdot)\|_{L^{2}\left(\mathbb{R}^{n} \backslash B\left(t^{1 / 2+\delta}\right)\right)},
$$

where we have used the fact that $j>0$, which implies (1.8).

To show Lemma2 we need a linear estimates for the fundamental solution of the following linear damped wave equation

$$
w_{t t}-\Delta w+w_{t}=0, \quad w(0, x)=u_{0}(x), \quad w_{t}(0, x)=u_{1}(x),
$$

for $t \in(0, \infty) \times \mathbb{R}^{n}$. Let $K_{0}(t), K_{1}(t)$ be

$$
K_{0}(t):=e^{-\frac{t}{2}} \cos \{t a(|\nabla|)\}, \quad K_{1}(t):=e^{-\frac{t}{2}} \frac{\sin \{t a(|\nabla|)\}}{a(|\nabla|)},
$$

where

$$
\mathcal{F}[a(|\nabla|)](\xi)=a(\xi)= \begin{cases}\sqrt{|\xi|^{2}-1 / 4}, & |\xi|>1 / 2, \\ i \sqrt{1 / 4-|\xi|^{2}}, & |\xi| \leq 1 / 2 .\end{cases}
$$

Note that $K_{0}(t)+1 / 2 K_{1}(t)=\partial_{t} K_{1}(t)$. Then the solution of (3.20) is given (cf. [17]) through the Fourier transform by $K_{0}(t)$ and $K_{1}(t)$ as

$$
w(t, x)=K_{0}(t) * u_{0}+K_{1}(t) *\left(\frac{1}{2} u_{0}+u_{1}\right) .
$$

The Duhamel principle implies that the solution $u(t, x)$ of nonlinear equation (1.1) solves the integral equation

$$
u(t, x)=w(t, x)+\Gamma(\alpha) \int_{0}^{t} K_{1}(t-\tau) * J_{0 \mid \tau}^{\alpha}\left(|u|^{p}\right)(\tau) d \tau,
$$

where $\alpha:=1-\gamma$ and $J_{0 \mid t}^{\alpha}$ is given by (2.2). We can now state Matsumura's result, on the estimate of $K_{0}(t)$ and $K_{1}(t)$, as follows: 
Lemma 4. ([1] $)$ If $f \in L^{m}\left(\mathbb{R}^{n}\right) \cap H^{k+|v|-1}\left(\mathbb{R}^{n}\right)(1 \leq m \leq 2)$, then

$$
\left\|\partial_{t}^{k} \nabla_{x}^{v} K_{1}(t) * f\right\|_{2} \leq C(1+t)^{n / 4-n /(2 m)-|v| / 2-k}\left(\|f\|_{m}+\|f\|_{H^{k+|| \mid-1}\left(\mathbb{R}^{n}\right)}\right) .
$$

Proof of Lemma 2, We begin to estimate the linear term $\|D w(t, \cdot)\|_{2}$. It is not difficult to see, using Lemma 4 with $m=1$, that

$$
\|D w(t, \cdot)\|_{2} \leq C(1+t)^{-n / 4-1 / 2}\left(\left\|u_{0}\right\|_{H^{1}}+\left\|u_{0}\right\|_{1}+\left\|u_{1}\right\|_{2}+\left\|u_{1}\right\|_{1}\right) \leq C I_{0}(1+t)^{-n / 4-1 / 2} \leq C I_{0}(1+t)^{-j} .
$$

To estimate the nonlinear term in (3.22), we have to distinguish two cases:

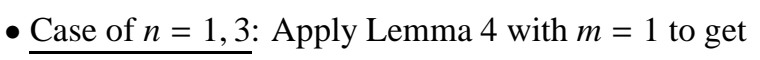

$$
\begin{aligned}
I:=\int_{0}^{t}\left\|D K_{1}(t-\tau) * J_{0 \mid \tau}^{\alpha}\left(|u|^{p}\right)(\tau)\right\|_{2} d \tau & \leq C \int_{0}^{t}(t-\tau+1)^{-n / 4-1 / 2}\left(\left\|J_{0 \mid \tau}^{\alpha}\left(|u|^{p}\right)(\tau)\right\|_{1}+\left\|J_{0 \mid \tau}^{\alpha}\left(|u|^{p}\right)(\tau)\right\|_{2}\right) d \tau \\
& \leq C \int_{0}^{t}(t-\tau+1)^{-n / 4-1 / 2}\left(J_{0 \mid \tau}^{\alpha}\|u(\tau)\|_{p}^{p}+J_{0 \mid \tau}^{\alpha}\|u(\tau)\|_{2 p}^{p}\right) d \tau .
\end{aligned}
$$

To transform the $L^{p}$-norm into a weighted $L^{2 p}$-norm, we use the Cauchy inequality

$$
\begin{aligned}
\|u(\tau, \cdot)\|_{p}^{p} & \equiv \int_{B(\tau+K)}|u(\tau, x)|^{p} d x \\
& \leq\left(\int_{B(\tau+K)} e^{-2 p \delta \psi(\tau, x)} d x\right)^{1 / 2}\left(\int_{B(\tau+K)} e^{2 p \delta \psi(\tau, x)}|u(\tau, x)|^{2 p} d x\right)^{1 / 2},
\end{aligned}
$$

for $\delta>0$. From (3.10), we have $\psi(\tau, x) \geq|x|^{2} / 4(\tau+K)$ for $x \in B(\tau+K)$, so the first integral is estimated as follows

$$
\int_{B(\tau+K)} e^{-2 p \delta \psi(\tau, x)} d x \leq \int_{B(\tau+K)} e^{-p \delta|x|^{2} / 2(\tau+k)} d x \leq \int_{\mathbb{R}^{n}} e^{-p \delta|x|^{2} / 2(\tau+k)} d x \equiv\left(\frac{2 \pi}{p \delta}\right)^{n / 2}(\tau+K)^{n / 2} .
$$

Thus, for the norm $\|u(\tau, \cdot)\|_{p}$ in (3.24) we obtain the weighted estimate

$$
\|u(\tau, \cdot)\|_{p}^{p} \leq C_{K, \delta}(\tau+1)^{n / 4}\left\|e^{\delta \psi(\tau, \cdot)} u(\tau, \cdot)\right\|_{2 p}^{p}, \quad \delta>0 .
$$

Next, as $\psi>0$, the norm $\|u(\tau, \cdot)\|_{2 p}$ in (3.24) can obviously be estimated by

$$
\|u(\tau, \cdot)\|_{2 p}^{p} \leq C_{\delta}(\tau+1)^{n / 4}\left\|e^{\delta \psi(\tau, \cdot)} u(\tau, \cdot)\right\|_{2 p}^{p} .
$$

Combining (3.24) - (3.26), we obtain

$$
\begin{aligned}
I & \leq C \int_{0}^{t}(t-\tau+1)^{-n / 4-1 / 2} \int_{0}^{\tau}(\tau-\sigma)^{-\gamma}\left((\sigma+1)^{n /(4 p)}\left\|e^{\delta \psi(\sigma, \cdot)} u(\sigma, \cdot)\right\|_{2 p}\right)^{p} d \sigma d \tau \\
& \leq C\left(\max _{[0, t]}(\tau+1)^{\beta}\left\|e^{\delta \psi(\tau,)} u(\tau, \cdot)\right\|_{2 p}\right)^{p} \int_{0}^{t}(t-\tau+1)^{-n / 4-1 / 2} \int_{0}^{\tau}(\tau-\sigma)^{-\gamma}(1+\sigma)^{-2(2-\gamma)} d \sigma d \tau .
\end{aligned}
$$

Using Lemma1, we conclude that

$$
I \leq C(1+t)^{-j}\left(\max _{[0, t]}(\tau+1)^{\beta}\left\|e^{\delta \psi(\tau, \cdot)} u(\tau, \cdot)\right\|_{2 p}\right)^{p} .
$$

Combining (3.23) and (3.27), we obtain (3.12). This complete the proof for $n=1,3$.

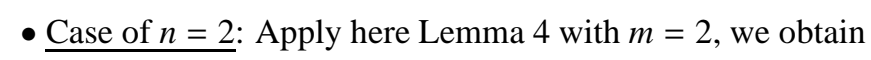

$$
\begin{aligned}
J:=\int_{0}^{t}\left\|D K_{1}(t-\tau) * J_{0 \mid \tau}^{\alpha}\left(|u|^{p}\right)(\tau)\right\|_{2} d \tau & \leq C \int_{0}^{t}(t-\tau+1)^{-1 / 2}\left\|J_{0 \mid \tau}^{\alpha}\left(|u|^{p}\right)(\tau)\right\|_{2} d \tau \\
& \leq C \int_{0}^{t}(t-\tau+1)^{-1 / 2} \int_{0}^{\tau}(\tau-\sigma)^{-\gamma}\|u(\sigma)\|_{2 p}^{p} d \sigma d \tau .
\end{aligned}
$$


Then

$$
J \leq C\left(\max _{[0, t]}(\tau+1)^{\beta}\left\|e^{\delta \psi(\tau, \cdot)} u(\tau, \cdot)\right\|_{2 p}\right)^{p} \int_{0}^{t}(t-\tau+1)^{-1 / 2} \int_{0}^{\tau}(\tau-\sigma)^{-\gamma}(1+\sigma)^{-2(2-\gamma)} d \sigma d \tau .
$$

By Lemma1, (3.28) implies

$$
J \leq C(1+t)^{-j}\left(\max _{[0, t]}(\tau+1)^{\beta}\left\|e^{\delta \psi(\tau, \cdot)} u(\tau, \cdot)\right\|_{2 p}\right)^{p} .
$$

Combining (3.23) and (3.29), we obtain (3.12). This complete the proof for $n=2$.

\section{Blow-up result}

In this section we devote ourselves to the proof of Theorem 2 We start by introducing the definition of the weak solution of (1.1).

Definition 1. (Weak solution) Let $T>0, \gamma \in(0,1)$ and $u_{0}, u_{1} \in L_{l o c}^{1}\left(\mathbb{R}^{n}\right)$. We say that $u$ is a weak solution if $u \in L^{p}\left((0, T), L_{l o c}^{p}\left(\mathbb{R}^{n}\right)\right)$ and satisfies

$$
\begin{aligned}
& \Gamma(\alpha) \int_{0}^{T} \int_{\mathbb{R}^{n}} J_{0 \mid t}^{\alpha}\left(|u|^{p}\right) \varphi d x d t+\int_{\mathbb{R}^{n}} u_{1}(x) \varphi(0, x) d x+\int_{\mathbb{R}^{n}} u_{0}(x)\left(\varphi(0, x)-\varphi_{t}(0, x)\right) d x \\
& =\int_{0}^{T} \int_{\mathbb{R}^{n}} u \varphi_{t t} d x d t-\int_{0}^{T} \int_{\mathbb{R}^{n}} u \varphi_{t} d x d t-\int_{0}^{T} \int_{\mathbb{R}^{n}} u \Delta \varphi d x d t,
\end{aligned}
$$

for all compactly supported function $\varphi \in C^{2}\left([0, T] \times \mathbb{R}^{n}\right)$ such that $\varphi(\cdot, T)=0$ and $\varphi_{t}(\cdot, T)=0$, where $\alpha=1-\gamma$.

Next, the following lemma is useful for the proof of Theorem 2 The proof of this lemma is much the same procedure as in the proof of [5, Lemma 2].

Lemma 5. (Mild $\rightarrow$ Weak) Let $T>0$ and $\gamma \in(0,1)$. Suppose that $1<p \leq n /(n-2)$, if $n \geq 3$, and $p \in(1, \infty)$, if $n=1,2$. If $u \in C\left([0, T], H^{1}\left(\mathbb{R}^{n}\right)\right) \cap C^{1}\left([0, T], L^{2}\left(\mathbb{R}^{n}\right)\right)$ is the mild solution of $[1.1]$, then $u$ is a weak solution of (1.1).

Remark. We need the mild solution to use, in the proof of Theorem 2 the alternative (1.6). Without this properties, we say that we have a nonexistence of global solution and not a blow-up result.

Proof of Theorem 2, We assume on the contrary, using (1.6), that $u$ is a global mild solution of (1.1). So, from Lemma[5] we have

$$
\begin{aligned}
& \Gamma(\alpha) \int_{0}^{T} \int_{\operatorname{supp} \varphi} J_{0 \mid t}^{\alpha}\left(|u|^{p}\right) \varphi d x d t+\int_{\operatorname{supp} \varphi} u_{1}(x) \varphi(0, x) d x+\int_{\operatorname{supp} \varphi} u_{0}(x)\left(\varphi(0, x)-\varphi_{t}(0, x)\right) d x \\
& =\int_{0}^{T} \int_{\operatorname{supp} \varphi} u \varphi_{t t} d x d t-\int_{0}^{T} \int_{\operatorname{supp} \varphi} u \varphi_{t} d x d t-\int_{0}^{T} \int_{\operatorname{supp} \Delta \varphi} u \Delta \varphi d x d t,
\end{aligned}
$$

for all $T>0$ and all compactly supported test function $\varphi \in C^{2}\left([0, T] \times \mathbb{R}^{n}\right)$ such that $\varphi(\cdot, T)=0$ and $\varphi_{t}(\cdot, T)=0$, where $\alpha=1-\gamma$. Let $\varphi(x, t)=D_{t \mid T}^{\alpha}(\tilde{\varphi}(x, t)):=D_{t \mid T}^{\alpha}\left(\varphi_{1}^{\ell}(x) \varphi_{2}(t)\right)$ with $\varphi_{1}(x):=\Phi(|x| / B), \varphi_{2}(t):=(1-t / T)_{+}^{\eta}$, where $D_{t \mid T}^{\alpha}$ is given by (2.1), $\ell, \eta \gg 1$ and $\Phi \in C^{\infty}\left(\mathbb{R}_{+}\right)$be a cut-off non-increasing function such that

$$
\Phi(r)= \begin{cases}1 & \text { if } 0 \leq r \leq 1 \\ 0 & \text { if } r \geq 2\end{cases}
$$

$0 \leq \Phi \leq 1$ and $\left|\Phi^{\prime}(r)\right| \leq C_{1} / r$ for all $r>0$. The constant $B>0$ in the definition of $\varphi_{1}$ is fixed and will be chosen later. In the following, we denote by $\Omega(B)$ the support of $\varphi_{1}$ and by $\Delta(B)$ the set containing the support of $\Delta \varphi_{1}$ which are defined as follows:

$$
\Omega(B)=\left\{x \in \mathbb{R}^{n}:|x| \leq 2 B\right\}, \quad \Delta(B)=\left\{x \in \mathbb{R}^{n}: B \leq|x| \leq 2 B\right\}
$$


We return to (4.2), which actually reads

$$
\begin{aligned}
& \Gamma(\alpha) \int_{0}^{T} \int_{\Omega(B)} J_{0 \mid t}^{\alpha}\left(|u|^{p}\right) D_{t \mid T}^{v} \tilde{\varphi} d x d t+\int_{\Omega(B)} u_{1}(x) D_{t \mid T}^{\alpha} \tilde{\varphi}(0, x) d x+\int_{\Omega(B)} u_{0}(x)\left(D_{t \mid T}^{\alpha} \tilde{\varphi}(0, x)-\partial_{t} D_{t \mid T}^{\alpha} \tilde{\varphi}(0, x)\right) d x \\
& =\int_{0}^{T} \int_{\Omega(B)} u \partial_{t}^{2} D_{t \mid T}^{\alpha} \tilde{\varphi} d x d t-\int_{0}^{T} \int_{\Omega(B)} u \partial_{t} D_{t \mid T}^{\alpha} \tilde{\varphi} d x d t-\int_{0}^{T} \int_{\Delta(B)} u \Delta D_{t \mid T}^{\alpha} \tilde{\varphi} d x d t .
\end{aligned}
$$

From (2.3), (2.4) and (2.7), we conclude that

$$
\begin{aligned}
& \int_{0}^{T} \int_{\Omega(B)} D_{0 \mid t}^{\alpha} J_{0 \mid t}^{\alpha}\left(|u|^{p}\right) \tilde{\varphi} d x d t+C T^{-\alpha} \int_{\Omega(B)} u_{1}(x) \varphi_{1}^{\ell}(x) d x+C\left(T^{-\alpha}+T^{-\alpha-1}\right) \int_{\Omega(B)} u_{0}(x) \varphi_{1}^{\ell}(x) d x \\
& =C \int_{0}^{T} \int_{\Omega(B)} u\left(D_{t \mid T}^{2+\alpha} \tilde{\varphi}+D_{t \mid T}^{1+\alpha} \tilde{\varphi}\right) d x d t-C \int_{0}^{T} \int_{\Delta(B)} u \Delta\left(\varphi_{1}^{\ell}\right) D_{t \mid T}^{\alpha} \varphi_{2} d x d t,
\end{aligned}
$$

where $D_{0 \mid t}^{\alpha}$ is defined in (2.1). Moreover, using (2.5) and the fact that (1.7) implies $\int_{\Omega(B)} \varphi_{1}^{\ell}(x) u_{i}(x) \geq 0, i=0,1$, it follows

$$
\begin{aligned}
\int_{0}^{T} \int_{\Omega(B)}|u|^{p} \tilde{\varphi} d x d t \leq & C \int_{0}^{T} \int_{\Omega(B)}|u| \varphi_{1}^{\ell}\left(D_{t \mid T}^{2+\alpha} \varphi_{2}+D_{t \mid T}^{1+\alpha} \varphi_{2}\right) d x d t \\
& +C \int_{0}^{T} \int_{\Delta(B)}|u| \varphi_{1}^{\ell-2}\left(\left|\Delta \varphi_{1}\right|+\left|\nabla \varphi_{1}\right|^{2}\right) D_{t \mid T}^{\alpha} \varphi_{2} d x d t \\
=: & I_{1}+I_{2},
\end{aligned}
$$

where we have used the formula $\Delta\left(\varphi_{1}^{\ell}\right)=\ell \varphi_{1}^{\ell-1} \Delta \varphi_{1}+\ell(\ell-1) \varphi_{1}^{\ell-2}\left|\nabla \varphi_{1}\right|^{2}$ and $\varphi_{1} \leq 1$. Next we observe that by introducing the term $\tilde{\varphi}^{1 / p} \tilde{\varphi}^{-1 / p}$ in the right side of (4.5) and applying Young's inequality we have

$$
I_{1} \leq \frac{1}{2 p} \int_{0}^{T} \int_{\Omega(B)}|u|^{p} \tilde{\varphi} d x d t+C \int_{0}^{T} \int_{\Omega(B)} \varphi_{1}^{\ell} \varphi_{2}^{-1 /(p-1)}\left(\left(D_{t \mid T}^{2+\alpha} \varphi_{2}\right)^{p^{\prime}}+\left(D_{t \mid T}^{1+\alpha} \varphi_{2}\right)^{p^{\prime}}\right) d x d t
$$

where $p^{\prime}=p /(p-1)$. Similarly,

$$
I_{2} \leq \frac{1}{2 p} \int_{0}^{T} \int_{\Omega(B)}|u|^{p} \tilde{\varphi} d x d t+C \int_{0}^{T} \int_{\Omega(B)} \varphi_{1}^{\ell-2 p^{\prime}} \varphi_{2}^{-1 /(p-1)}\left(\left|\Delta \varphi_{1}\right|^{p^{\prime}}+\left|\nabla \varphi_{1}\right|^{2 p^{\prime}}\right)\left(D_{t \mid T}^{\alpha} \varphi_{2}\right)^{p^{\prime}} d x d t .
$$

Combining (4.6) and (4.7), it follows from (4.5) that

$$
\begin{aligned}
\int_{0}^{T} \int_{\Omega(B)}|u|^{p} \tilde{\varphi} d x d t \leq & C \int_{0}^{T} \int_{\Omega(B)} \varphi_{1}^{\ell} \varphi_{2}^{-1 /(p-1)}\left(\left(D_{t \mid T}^{2+\alpha} \varphi_{2}\right)^{p^{\prime}}+\left(D_{t \mid T}^{1+\alpha} \varphi_{2}\right)^{p^{\prime}}\right) d x d t \\
& +C \int_{0}^{T} \int_{\Omega(B)} \varphi_{1}^{\ell-2 p^{\prime}} \varphi_{2}^{-1 /(p-1)}\left(\left|\Delta \varphi_{1}\right|^{p^{\prime}}+\left|\nabla \varphi_{1}\right|^{2 p^{\prime}}\right)\left(D_{t \mid T}^{\alpha} \varphi_{2}\right)^{p^{\prime}} d x d t .
\end{aligned}
$$

At this stage, to prove $i$ ), we have to distinguishes 2 cases.

- Case of $p<p_{\gamma}$ : in this case, we take $B=T^{1 / 2}$. So, using (2.6) and the change of variables: $s=T^{-1} t, y=T^{-1 / 2} x$, we get from (4.8) that

$$
\int_{0}^{T} \int_{\Omega\left(T^{1 / 2}\right)}|u|^{p} \tilde{\varphi} d x d t \leq C\left(T^{-(\alpha+2) p^{\prime}+n / 2+1}+T^{-(\alpha+1) p^{\prime}+n / 2+1}\right),
$$

where $C$ is independent of $T$. Letting $T \rightarrow \infty$ in (4.9), thanks to $p<p_{\gamma}$ and the Lebesgue dominated convergence theorem, it is yielded that

$$
\int_{0}^{\infty} \int_{\mathbb{R}^{n}}|u|^{p} d x d t=0
$$


which implies $u(x, t)=0$ for all $t$ and a.e. $x$. This contradicts our assumption 1.77 .

- Case of $p=p_{\gamma}$ : let $B=R^{-1 / 2} T^{1 / 2}$, where $1 \ll R<T$ is such that when $T \rightarrow \infty$ we don't have $R \rightarrow \infty$ at the same time. Moreover, from the last case and the fact that $p=p_{\gamma}$, there exist a positive constant $D$ independent of $T$ such that

$$
\int_{0}^{\infty} \int_{\mathbb{R}^{n}}|u|^{p} d x d t \leq D
$$

which implies that

$$
\int_{0}^{T} \int_{\Delta\left(R^{-1 / 2} T^{1 / 2}\right)}|u|^{p} \tilde{\varphi} d x d t \rightarrow 0 \quad \text { as } T \rightarrow \infty .
$$

On the other hand, using Hölder's inequality instead of Young's one, we estimate the integral $I_{2}$ in (4.5) as follows:

$$
I_{2} \leq C\left(\int_{0}^{T} \int_{\Delta\left(R^{-1 / 2} T^{1 / 2}\right)}|u|^{p} \tilde{\varphi}\right)^{1 / p}\left(\int_{0}^{T} \int_{\Omega\left(R^{-1 / 2} T^{1 / 2}\right)} \varphi_{1}^{\ell-2 p^{\prime}} \varphi_{2}^{-1 /(p-1)}\left(\left|\Delta \varphi_{1}\right|^{p^{\prime}}+\left|\nabla \varphi_{1}\right|^{2 p^{\prime}}\right)\left(D_{t \mid T}^{\alpha} \varphi_{2}\right)^{p^{\prime}} d x d t\right)^{1 / p^{\prime}} .
$$

Similarly to the last case, substituting (4.6) and (4.11) into (4.5), taking account of $p=p_{\gamma}$ and the scaled variable $s=T^{-1} t, y=R^{1 / 2} T^{-1 / 2} x$, we get

$$
\int_{0}^{T} \int_{\Omega\left(R^{-1 / 2} T^{1 / 2}\right)}|u|^{p} d x d t \leq C\left(T^{-p^{\prime}} R^{-n / 2}+R^{-n / 2}\right)+C R^{1-n /\left(2 p^{\prime}\right)}\left(\int_{0}^{T} \int_{\Delta\left(R^{-1 / 2} T^{1 / 2}\right)}|u|^{p} \tilde{\varphi}\right)^{1 / p} .
$$

Letting $T \rightarrow \infty$, using (4.10), we get

$$
\int_{0}^{\infty} \int_{\mathbb{R}^{N}}|u|^{p} d x d t \leq C R^{-n / 2}
$$

which implies a contradiction, when $R \rightarrow \infty$, with (1.7). This completes the proof of Theorem $2, i$ ).

For the proof of $i i)$, we have two possibility.

- If $\gamma<(n-2) / n$ : let $B=R$ with the same $R$ introduced in the case $p=p_{\gamma}$. Then, taking the scaled variables $s=T^{-1} t, y=R^{-1} x$, it follows from (4.8) that

$$
\int_{0}^{T} \int_{\Omega(R)}|u|^{p} \tilde{\varphi} d x d t \leq C R^{n}\left(T^{-(2+\alpha) p^{\prime}+1}+T^{-(1+\alpha) p^{\prime}+1}\right)+C R^{n-2 p^{\prime}} T^{-\alpha p^{\prime}+1} .
$$

As $\gamma<(n-2) / n$ implies $p \leq n / n-2<1 / \gamma$, we get a contradiction with (1.7) by letting the following limits: first $T \rightarrow \infty$, next $R \rightarrow \infty$.

- If $\gamma=(n-2) / n$ : we have $p \leq n /(n-2)=1 / \gamma=p_{\gamma}$. Using the first two cases, we get the contradiction. This completes the proof of Theorem [2, ii).

\section{Appendix A.}

In this appendix let us sketch the proof of Proposition 1, Let us define a semigroup $S(t): H^{1}\left(\mathbb{R}^{n}\right) \times L^{2}\left(\mathbb{R}^{n}\right) \rightarrow$ $H^{1}\left(\mathbb{R}^{n}\right) \times L^{2}\left(\mathbb{R}^{n}\right)$ by

$$
S(t):\left[\begin{array}{l}
u_{0} \\
u_{1}
\end{array}\right] \mapsto\left[\begin{array}{c}
w \\
w_{t}
\end{array}\right],
$$

where $w \in C\left([0, \infty), H^{1}\left(\mathbb{R}^{n}\right)\right) \cap C^{1}\left([0, \infty), L^{2}\left(\mathbb{R}^{n}\right)\right)$ is the linear solution of (3.20) given by (3.21). So, view of (3.22), a mild solution of the nonlinear problem (1.1) is equivalent to following integral equation:

$$
U(t)=S(t) U_{0}+\int_{0}^{t} S(t-s) F(s) d s,
$$


where

$$
U(t)=\left[\begin{array}{c}
u(t, \cdot) \\
u_{t}(t, \cdot)
\end{array}\right], \quad U_{0}=\left[\begin{array}{l}
u_{0} \\
u_{1}
\end{array}\right], \quad F(s)=\left[\begin{array}{l}
0 \\
J_{0 \mid s}^{\alpha}\left(|u|^{p}\right)(s)
\end{array}\right] .
$$

It sufficient now to prove the local existence of a solution of A.1 in $H^{1}\left(\mathbb{R}^{n}\right) \times L^{2}\left(\mathbb{R}^{n}\right)$. Let $T>0$ and consider the following Banach space

$$
E:=\left\{U={ }^{t}(u, v):(u, v) \in C\left([0, T], H^{1}\left(\mathbb{R}^{n}\right) \times L^{2}\left(\mathbb{R}^{n}\right)\right), \operatorname{supp} u(t, \cdot) \subset B(K+t) \text { and }\|U\|_{E} \leq C M\right\},
$$

where

$$
\|U\|_{E}:=\|u\|_{C\left([0, T] ; H^{1}\left(\mathbb{R}^{n}\right)\right)}+\|v\|_{C\left([0, T] ; L^{2}\left(\mathbb{R}^{n}\right)\right)} \quad \text { and } \quad M:=\left\|u_{0}\right\|_{H^{1}}+\left\|u_{1}\right\|_{2} .
$$

In order to use the Banach fixed point theorem, we introduce the following map $\Phi$ on $E$ defined by

$$
\Phi[U](t):=S(t) U_{0}+\int_{0}^{t} S(t-s) F(s) d s
$$

Now, for $U=(u, v) \in E$, we have

$$
\left\|J_{0 \mid t}^{\alpha}\left(|u|^{p}\right)(t)\right\|_{2} \leq C t^{1-\gamma}\|u(t, \cdot)\|_{2 p}^{p} \leq C t^{1-\gamma}\|u(t, \cdot)\|_{H^{1}}^{p} \leq C t^{1-\gamma}\|U\|_{E}^{p}, \quad t \in[0, T],
$$

where we have used the Sobolev imbedding $H^{1}\left(\mathbb{R}^{n}\right) \subset L^{2 p}\left(\mathbb{R}^{n}\right)$. Next, using Matsumura's result (Lemma 4) with $m=2$ and the finite propagation speed phenomena, we deduce via the Banach fixed point theorem that there exists a local solution $U \in E$ on a small interval $[0, T]$ satisfies A.1. For details, we refer the reader to [7, Theorem 3.2] and [5, Theorem 6]. By consequence, there exist a local solution $u \in C\left([0, T], H^{1}\left(\mathbb{R}^{n}\right)\right) \cap C^{1}\left([0, T], L^{2}\left(\mathbb{R}^{n}\right)\right)$ satisfies (3.22) and $\operatorname{supp} u(t, \cdot) \subset B(t+K)$. However, since our equation (1.1) is nonautonomous, we prefer apply Gronwall's inequality to get the uniqueness (cf. [2, Theorem 3.1]). Indeed, if $u, v \in C\left([0, T], H^{1}\left(\mathbb{R}^{n}\right)\right) \cap C^{1}\left([0, T], L^{2}\left(\mathbb{R}^{n}\right)\right)$ are two mild solutions (i.e. satisfy (3.22) for some $T>0$, we have

$$
\begin{aligned}
\|u(t)-v(t)\|_{H^{1}} & \leq C \int_{0}^{t}\left\|K_{1}(t-\tau) * J_{0 \mid \tau}^{\alpha}\left(|u|^{p}-|v|^{p}\right)(\tau)\right\|_{H^{1}} d \tau \\
& \leq C \int_{0}^{t}(1+t-\tau)^{-1 / 2}\left\|J_{0 \mid \tau}^{\alpha}\left(|u|^{p}-|v|^{p}\right)(\tau)\right\|_{2} d \tau \\
& \leq C \int_{0}^{t}\left\|J_{0 \mid \tau}^{\alpha}\left(|u|^{p}-|v|^{p}\right)(\tau)\right\|_{2} d \tau,
\end{aligned}
$$

where we have used again Matsumura's result (Lemma 4) with $m=2$. As $\|\left. u\right|^{p}-|v|^{p}|\leq C| u-v \mid\left(|u|^{p}+|v|^{p}\right.$ ), so by Hölder's inequality $\left(\|a b\|_{2} \leq\|a\|_{2 p}\|b\|_{2 p^{\prime}}\right)$ with $p^{\prime}=p /(p-1)$ and Sobolev's imbedding $\left(H^{1} \subset L^{2 p}\right)$, we obtain

$$
\begin{aligned}
\int_{0}^{t}\left\|J_{0 \mid \tau}^{\alpha}\left(|u|^{p}-|v|^{p}\right)(\tau)\right\|_{2} d \tau & \leq C \int_{0}^{t} J_{0 \mid \tau}^{\alpha}\left(\|u-v\|_{H^{1}}\left(\|u\|_{H^{1}}^{p-1}+\|v\|_{H^{1}}^{p-1}\right)\right)(\tau) d \tau \\
& \leq C \int_{0}^{t} \int_{0}^{\tau}(\tau-s)^{-\gamma}\|u(s, \cdot)-v(s, \cdot)\|_{H^{1}} d s d \tau \\
& =C \int_{0}^{t} \int_{s}^{t}(\tau-s)^{-\gamma}\|u(s, \cdot)-v(s, \cdot)\|_{H^{1}} d \tau d s \\
& =C \int_{0}^{t}(t-s)^{1-\gamma}\|u(s, \cdot)-v(s, \cdot)\|_{H^{1}} d s .
\end{aligned}
$$

Combining (A.2) and , we get

$$
\|u(t)-v(t)\|_{H^{1}} \leq C \int_{0}^{t}(t-s)^{1-\gamma}\|u(s, \cdot)-v(s, \cdot)\|_{H^{1}} d s .
$$

Using Gronwall's inequality, it follows that $u(t) \equiv v(t)$. As a consequence of this uniqueness result, we can extend our solution $u$ on a maximal interval $\left[0, T_{\max }\right)$. Moreover, if $T_{\max }<\infty$, then $\|u(t, \cdot)\|_{H^{1}}+\left\|u_{t}(t, \cdot)\right\|_{2} \rightarrow \infty$ as $t \rightarrow T_{\max }$. For details, see [2, Theorem 3.1] and [7, Theorem 3.2]. 


\section{Acknowledgements}

The author was supported by the Lebanese Association for Scientific Research (LASeR). The author would like to express sincere gratitude to Professor Mokhtar Kirane for valuable discussion. He thanks Professors Vladimir Georgiev, Thierry Cazenave and Flavio Dickstein for their helful remarks.

\section{References}

[1] H. Bellout, A. Friedman, Blow-up estimates for a nonlinear hyperbolic heat equation, Math. Z. 145 (1975), 251 - 254.

[2] T. Cazenave, F. Dickstein, F. D. Weissler, An equation whose Fujita critical exponent is not given by scaling, Nonlinear Analysis 68 (2008), $862-874$.

[3] S. Cui, Local and global existence of solutions to semilinear parabolic initial value problems, Nonlinear Analysis 43 (2001), 293 - 323.

[4] L. Debnath, D. Bhatta, Integral Transforms and Their Applications, Taylor and Francis group, 2007.

[5] A. Z. Fino, V. Georgiev, M. Kirane, Finite time blow-up for a wave equation with a nonlocal nonlinearity, submitted.

[6] A. Fino, G. Karch, Decay of mass for nonlinear equation with fractional Laplacian, J. Monatsh. Math. 160 (2010), 375 - 384.

[7] A. Z. Fino, M. Kirane, Qualitative Properties of Solutions to a Time-Space Fractional Evolution Equation, to appear in Quarterly of Applied Mathematics.

[8] H. Fujita, On the blowing up of solutions of the problem for $u_{t}=\Delta u+u^{1+\alpha}$, J. Fac. Sci. Univ. Tokyo 13 (1966), $109-124$.

[9] T. Hosono, T. Ogawa, Large time behavior and $L^{p}-L^{q}$ estimate of solutions of 2-dimensional nonlinear damped wave equations, J. Differential Equations 203 (2004), $82-118$.

[10] R. Ikehata, Y. Miyaoka, T. Nakatake, Decays estimates of solutions for dissipative wave equation in $\mathbb{R}^{n}$ with lower power nonlinearities, J. Math. Soc. Japan 56 (2004), 365 - 373.

[11] R. Ikehata, K. Tanizawa, Global existence for solutions for semilinear damped wave equation in $\mathbb{R}^{N}$ with noncompactly supported initial data, Nonlinear Analysis 61 (2005), 1189 - 1208.

[12] G. Karch, Selfsimilar profiles in large time asymptotics of solutions to damped wave equations , Studia Math. 143 (2000), 175 - 197.

[13] A. A. Kilbas, H. M. Srivastava, J. J. Trujillo, Theory and Applications of Fractional Differential Equations, 2006.

[14] M. Guedda, M. Kirane, Criticality for some evolution equations, Differential Equations 37 (2001), $511-520$.

[15] M. Kirane, Y. Laskri, N.-e. Tatar, Critical exponents of Fujita type for certain evolution equations and systems with Spatio-Temporal Fractional derivatives, J. Math. Anal. Appl. 312 (2005), 488 - 501.

[16] T.-T. Li, Nonlinear heat conduction with finite speed of propagation, Proceedings of the China-Japan Symposium on Reaction-Diffusion Equations and their Applications and Computational Aspect, Shanghai, 1994, World Scientific Publishing, River Edge, NJ, (1997), 81 - 91.

[17] A. Matsumura, On the asymptotic behavior of solutions of semi-linear wave equations , Publ. RIMS Kyoto Univ. 121 (1976), 169 - 189.

[18] E. Mitidieri, S. I. Pohozaev, A priori estimates and blow-up of solutions to nonlinear partial differential equations and inequalities, Proc. Steklov. Inst. Math. 234 (2001), 1 - 383.

[19] E. Mitidieri, S. I. Pohozaev, Nonexistence of weak solutions for some degenerate elliptic and parabolic problems on $\mathbb{R}^{N}$, J. Evol. Equ. 1 (2001), $189-220$.

[20] T. Narazaki, $L^{p}-L^{q}$ estimates for damped wave equations and their applications to semi-linear problem, J. Math. Soc. Japan 56 (2004), $585-626$.

[21] K. Nishihara, $L^{p}-L^{q}$ estimates of solutions to the damped wave equation in 3-dimensional space and their application, Math. Z. 244 (2003), $631-649$.

[22] K. Nishihara, H. Zhao, Decay properties of solutions to the Cauchy problem for the damped wave equation with absorption, J. Math. Anal. Appl. 313 (2006), $598-610$.

[23] S. G. Samko, A. A. Kilbas, O. I. Marichev, Fractional integrals and derivatives, Theory and Applications, Gordon and Breach Science Publishers, 1987

[24] G. Todorova, B. Yordanov, Critical exponent for a nonlinear wave equation with damping, J. Differential Equations 174 (2001), 464 - 489.

[25] Qi S. Zhang, A blow up result for a nonlinear wave equation with damping: the critical case, C. R. Acad. Sci. Paris, Vol. 333 (2001), no. 2, $109-114$. 